\title{
MAXWELL'S FIRST COLOURED LIGHT SOURCES: ARTISTS' PIGMENTS
}

\author{
R.C. DOUGAL \\ James Clerk Maxwell Foundation, Scotland, UK.
}

\begin{abstract}
A description is given of James Clerk Maxwell's strategy, in his early study of the additive mixing of light from coloured samples. He used the scattered daylight from known areas of card coated with artists' pigments. Vermilion, emerald green and ultramarine were the optimum choice of standards for red, green and blue, respectively. They suited Thomas Young's description of colour vision. Maxwell's design of an analogue device - his 'colour top' - for varying the areas of the contributing pigments was remarkably simple. His meticulous observations with it allowed him to substantially further the understanding of perception of colour at the time, mid-19th century. The interpretation of a few very basic spectroscopic measurements on sunlight reflected from pigments are in line with Maxwell's conclusions.

Keywords: additive colour mixing of light, artists' pigments, colour top, spectra.
\end{abstract}

\section{INTRODUCTION}

Maxwell's earliest research on the perception of colour used daylight reflected from artists' pigments. By observing in broadly repeatable ambient conditions, he was able to identify regularities in the perception of combinations of light reflected from the range of colours provided by these pigments. He was guided in his design of equipment and strategy by the description of Thomas Young [1] of the most likely structure of those light sensitive detectors in the human eye - cells called 'cones' because of their shape - which are sensitive to colour. Coloured image production, especially projected images but also in colour photography [2], owes much to Maxwell's researches. So does the use of colour in the design of objects across the whole range of manufacturing industry, and in the design of light sources.

\subsection{Apparatus}

\section{MAXWELL'S STRATEGY}

Maxwell's apparatus [3] - his colour top - was a step change in design from the preceding work of James D. Forbes, Professor of Natural Philosophy at Edinburgh University, where Maxwell was a student from 1847 to 1850 . His work on colour continued when he transferred to Cambridge University in 1850 (Fig. 1).

On to a circular platform approximately $20 \mathrm{~cm}$ in diameter, Maxwell placed three interleaved coloured discs - card painted with artists' pigments as indicated in Fig. 2. The areas of the exposed segments could be varied, by hand, continuously and then screwed fixed while the top was spun. The perimeter scale, calibrated from 0 to 100 , provided directly the relative areas of the exposed segments. The two smaller interleaved discs mounted centrally allowed systematic comparative measurements to be made for the range of pigments chosen [3].

When the disc was spun, each segment swept across, appearing to cover, the whole area of the platform (Fig. 3). The eye therefore intercepted simultaneously light from the three pigments propagating independently between the circular area of the top's platform and observer. The spectrum of the light arriving at the eye was a combination of the three spectra from the pigments used. The response of the eye-brain system was the perceived colour of the composite object. 

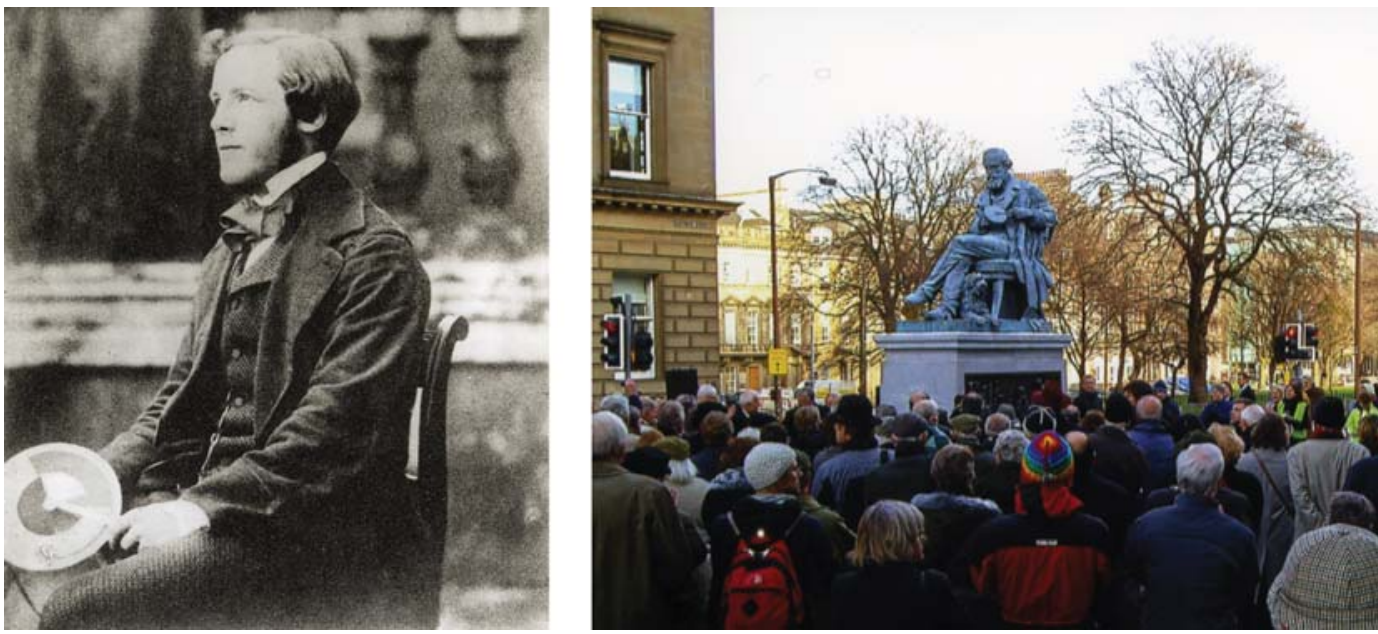

Figure 1: Maxwell as a young man at Cambridge (ca. 1854), holding the colour top. Reproduced by permission of the Master and Fellows of Trinity College Cambridge (left). Statue (by Alexander Stoddart) of James Clerk Maxwell, unveiled in George Street, Edinburgh on 25 November, 2008. It shows Maxwell in period dress, holding in his hands his colour top (right).
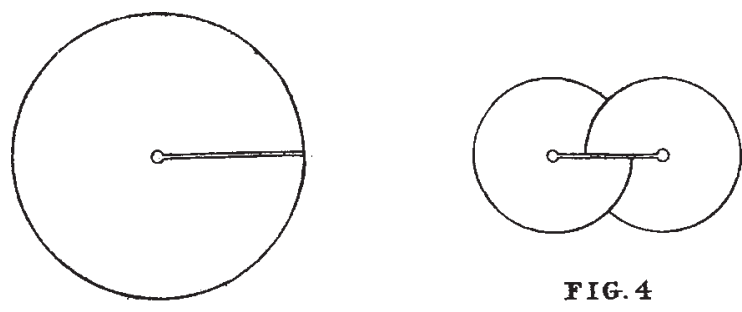

F IG. 4

FIG.3

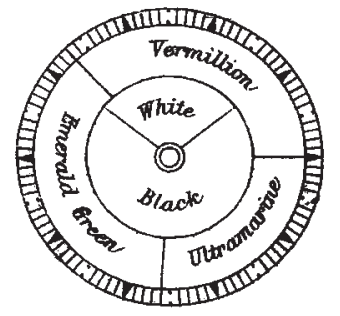

F I G. $\boldsymbol{b}$

Figure 2: Drawing showing design of layout of coloured segments on Maxwell's colour top. Reproduced by permission of the Royal Society of Edinburgh from Transactions of the Royal Society of Edinburgh, volume XXI, pp. 275-298, 1853-1857. 

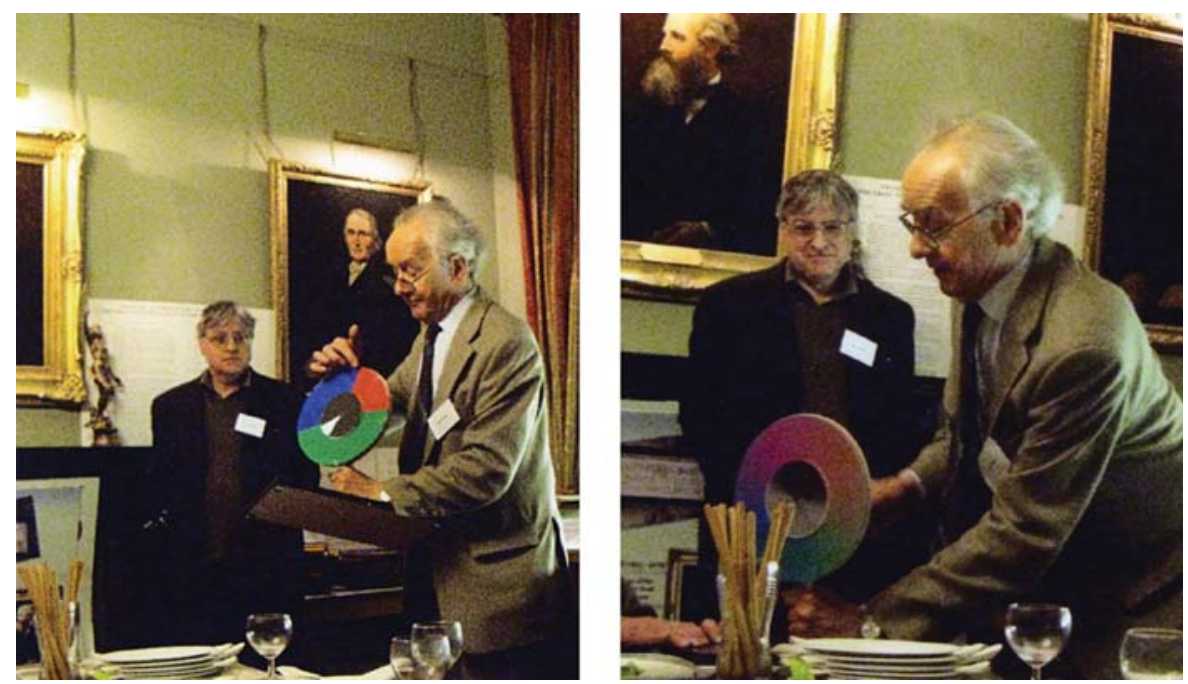

Figure 3: Demonstration model of Maxwell's colour top being shown following the evening. Reception at Maxwell's birthplace, 14 India Street, Edinburgh (left). Demonstration top being spun. Fortuitously, the rotation speed and exposure time permitted imaging together of light from adjacent colour segments. Visible are the brown from green and red, and the magenta from red and blue. The cyan from blue and green is obscured. Photographs courtesy of Madeleine Shepherd.

\subsection{Observations}

Young had pointed out that there had to be light sensitive areas on the retina at the back of the eye which responded to different regions of the visible spectrum. Three such areas were sufficient, sensitive to different colour ranges: violets and blues (least sensitive); the whole spectrum but especially the greens and neighbouring blues and yellows; and the whole spectrum but especially yellow and the neighbouring greens and reds - the last, curiously, including a relatively slight enhanced sensitivity to blue and violet at the other end of the spectrum. The areas are networks of light sensitive cells called 'cones' because of their shape. The areas are now referred to as preferentially sensitive to short (S), medium (M) and long (L) wavelengths. All three areas responded to the 'white light' of daylight. If all could respond similarly to the composite light from red, green and blue pigments, the observer would 'see' a colour depending on the proportions of red, green and blue on the platform. Selective reflection of the light of the complete daylight spectrum implies selective absorption. The colour from one unique set of proportions would be untinted, or neutral, grey rather than the white of (nearly) the full daylight spectrum that would be reflected from best quality white card.

Maxwell's strategy was to match that grey to the grey that was produced by the light from two smaller interleaved discs, one white and the other black, mounted centrally on the platform of the top. His optimum choices of red, green and blue pigments as standards were vermilion, emerald green and ultramarine, respectively. A few other combinations of pigments could also produce other shades of grey. From the tallies of the sets of five reflecting areas, three for pigments and one each for white and black, Maxwell was able to describe the colours of any of the other pigments in terms 

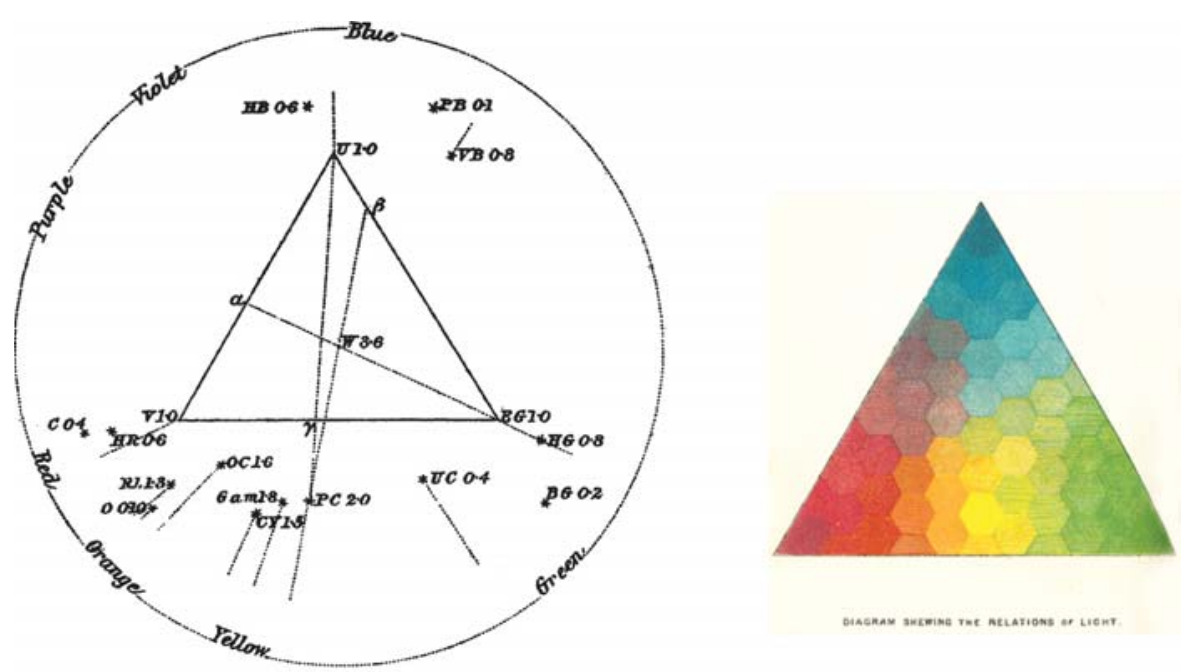

(N:1)

Figure 4: Maxwell's diagram with the positions of the various pigments marked in relative to the positions of the standards, got from observations of the light scattered by the various pigments (left). Reproduced by permission of the Royal Society of Edinburgh from Transactions of the Royal Society of Edinburgh, volume XXI, pp. 275-298, 1853-1857. Maxwell's colour triangle [5] (right).

of his standards. On to Newton's circle of colours [4], he superimposed an equilateral triangle of colours (Fig. 4). Alongside is Maxwell's sketch of his colour triangle [5].

The colour of each of his three standard pigments (vermilion, emerald green and ultramarine) is given 'weight' 1 . Suppose light is obtained from known proportions of the three pigments, giving their relative 'weights'. Following Newton, the position of the corresponding colour might be expected to lie somewhere in the triangle, got from a centre of gravity type of estimate. In Fig. 5, from [5], is a diagrammatic summary of Maxwell's results. The positions for other pigments lie outside the triangle but are keyed geometrically to the colours inside. The numbers alongside indicate their weights. The 1.3 for pale chrome shows that it was brighter than the vermilion, consistent with the observation that when vermilion was replaced by pale chrome, the matching grey was lighter. He thus established that basic rules for combining light from different pigments (additive mixing) could be written in terms of vermilion, emerald green and ultramarine blue as the primaries. That pale chrome and the others should lie outside the triangle was an outcome from Maxwell's analysis of the relevant tallies in the records of his observations. They led to proportions of pale chrome and ultramarine from which the perceived colour would match unique proportions of vermilion and emerald green. They also pointed to yellows and oranges lying between greens and reds.

The observations were extended to match colours rather than two greys. His strategy [3] was far removed from trial and error:

Thus we may combine ultramarine, pale chrome and black, so as to produce a tint [in the annulus] identical with that of a compound of vermilion and emerald green [in the centre] .... The best method of arriving at a result in the case before us, is to render the hue of the red and the green 
combination something like that of the yellow, to reduce the purity of the yellow by the admixture of blue, and to diminish the intensity by the addition of black.

Yet a further extension was to match the tints when observing them through coloured glass and under different illuminations. Of the latter, there were only two of any use - 'day-light' and 'gaslight (Edinburgh)'. The proportions of the pigments had to be readjusted each time. Maxwell commented that all 'these experiments are really evidence relating to the constitution of eye, and not mere comparisons of two things which are in themselves identical ...'.

By contrast, red, yellow and blue are the primary colours of artists. Light from a patch painted as any mixture of red, yellow and blue pigments is intercepted by the eye as a single beam whose spectrum is interpreted by the eye as a single colour. The colour for artists' pigments so mixed is called 'subtractive': the colour we see is the response to composite light having those wavelengths not absorbed by the pigment mix.

\subsection{Interpretation}

The boundary of Maxwell's colour triangle can be superimposed on to the CIE chromaticity chart (Fig. 5), matching approximately the colours of his artists' pigments - to which we no longer have access - to three of the colours on the chart [6]. This confirms that the gamut of colours got from combining light from various proportions of vermilion, emerald green and ultramarine is only a fraction of the complete range including other pigments. This is how the gamuts of available colours from other choices of standards can still be conveniently represented diagrammatically nowadays (see e.g. Meyn [7]).

The observations required dedication, even doggedness, on the part of the team. This was typically Maxwell in control of the equipment and the volunteer observer, trained to keep his eyes relaxed by looking only at the spinning top and then only when directed to do so by Maxwell, and
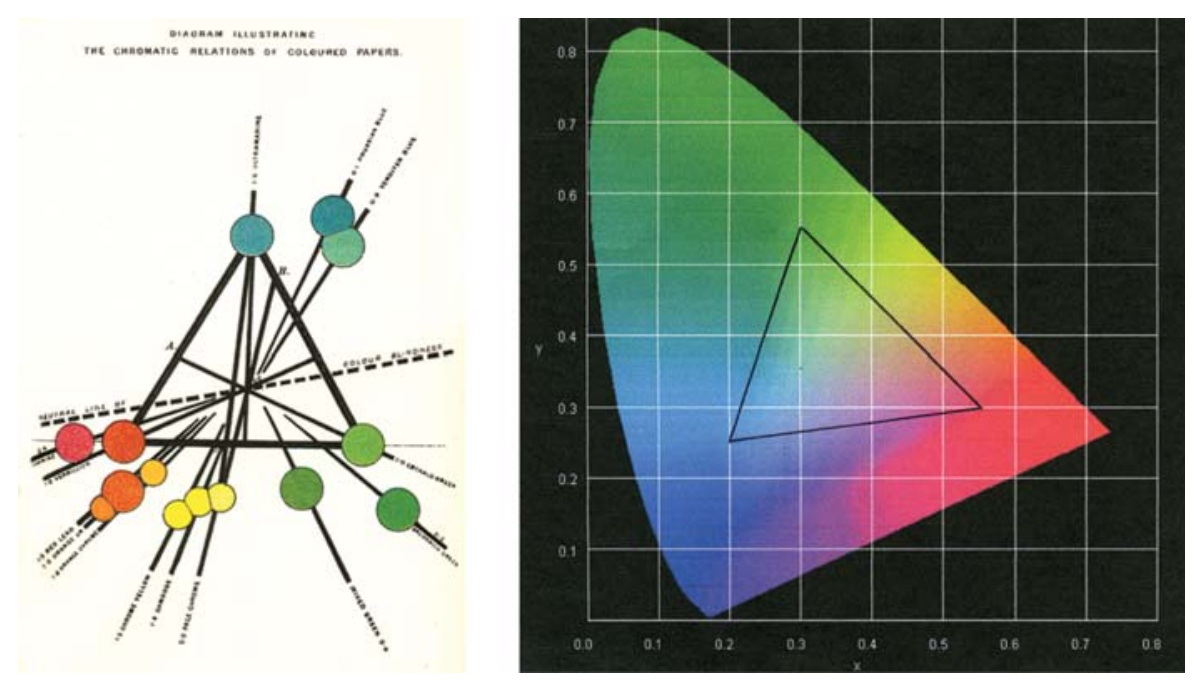

Figure 5: Maxwell's display of the results of his observations [5] (left). CIE chromaticity chart with estimate of position of Maxwell's colour triangle superimposed. Chart from website of Colour Group (Great Britain) [6]. 
describing any mismatches (e.g. 'too dark', 'too green') until the areas of the pigments had been adjusted to produce a perfect match between the central disk and the annular area surrounding it.

Maxwell found very little variation in the response to colour of his group of observers. On the way, he found a few, who were colour blind, and with their cooperation he made some of the very earliest semi-quantitative studies of such deficiencies - an interest he maintained throughout his life.

\section{OBSERVATIONS AND ANALYSIS USING MODERN PIGMENTS}

The option of recording spectra rapidly makes it possible to check out some of Maxwell's conclusions. Here, we report on the spectra of sunlight (late morning, October 21, 2008, Edinburgh) reflected by colour samples. The spectra of light recorded by a spectrophotometer type EO- 85 by the Daedelon Corporation, USA show the selectivity of modern pigments vermilion, emerald green and ultramarine pigments (Fig. 6 and Table 1). The same areas of pigments were painted inexpertly directly on to white card. Direct sunlight reflected in sequence from an equal area (circular, diameter $10.5 \mathrm{~cm}$ ) of unpainted card and then from three painted samples was focused through the entrance aperture of the device, using a lens of focal length $5 \mathrm{~cm}$. The spectrophotometer's diffraction grating directed the light into different directions according to its wavelength. The diffracted radiation is intercepted by a strip of approximately 1700 pixels in a narrow silicon layer - rather like a one dimensional digital camera. The output from the pixels, plotted as a graph, is only approximately proportional to the intensities of the component beams they intercept. The sensitivity of the silicon increases with wavelength. The graph overestimates the intensities progressively from the violet end of the spectrum to the infrared (wavelengths greater than about $700 \mathrm{~nm}$ ). The graphs are representations of what is in effect the particular perception of the colours by the spectrophotometer. Apart from this non-linearity in response, a small background signal across the graph arises from background radiation scattered into the spectrophotometer aperture from the sunlit laboratory, in which only minimal precautions to
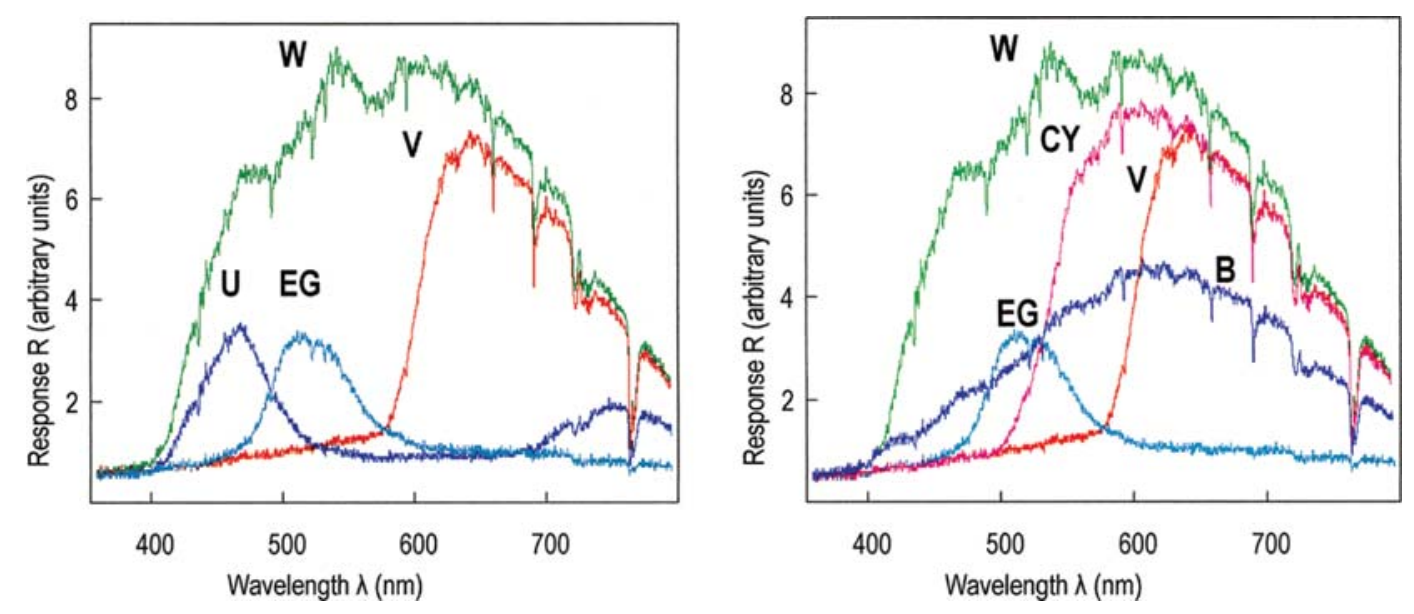

Figure 6: Spectra of light from equal circular areas cut from white card (W), and from three equal circular areas of the same card coated with artists' pigments vermilion (V), emerald green (EG) and ultramarine (U) (left). Spectra of light from equal circular areas cut from white card (W), and from four equal circular areas of the same card coated with artists' pigments vermilion (V), chrome yellow (CY), emerald green (EG) and of a buff envelope (B) (right) (Table 1). 
Table 1: Artists' pigments [Daler-Rowney (Georgian)] used to provide reflected light for spectroscopic analysis.

\begin{tabular}{lll}
\hline Pigment & \multicolumn{1}{c}{ Components } & \multicolumn{1}{c}{ Codes } \\
\hline Vermilion (hue) 588 & Arylamide yellow & GX PY73 \\
& Disazo orange & PO 34 \\
& BON arylamide red & PR 210 \\
Emerald Green (hue) & Arylamide yellow & IOGPY3 \\
& Phthalocyanine green & PG7 \\
French ultramarine 123 & Zinc oxide & PW4 \\
Chrome yellow (hue) 623 & Ultramarine blue & PB 29 \\
& Arylamide yellow & PY 1:1 \\
& Arylamide yellow & GX PY 73 \\
\hline
\end{tabular}

suppress background are possible (and justified for this purpose). Each pigment produces its characteristic spectrum of wavelengths of the radiation it selectively reflects. The white card reflects all wavelengths present. It is the solar spectrum in which the many dips result from absorption of radiation in its path between the sun and the earth - the so-called Fraunhofer absorption lines.

Maxwell's observations in [3] referred to above together with the appearance of the demonstration top spinning (Fig. 3) indicate that a suitably weighted combination of light from vermilion and emerald green pigments should produce the sensation of brown. Taking advantage of the bright mid-morning sunlight on the day, the measurements reported here had been extended to include the spectrum of light reflected from a circular disc cut from a pale brown (buff) envelope, and from the artists' pigment chrome yellow (Fig. 6). The spectra peak at very nearly the same wavelength. Simplifying, the brown can be thought of as a darkened (or less bright) yellow. However, the brown spectrum extends further into the shorter wavelengths. A glance at the spectra for vermilion and emerald green does suggest that from them a composite spectrum for some shade of brown should result. This is in line with the spectroscopic measurements by Erjavac and Vaupotic [8] on the perceived brown of dyes and leaves.

\section{CONCLUSION}

The path from Maxwell's observations of light from artists' pigments to current research in colour science is not direct. He progressed to working with light from sunlight dispersed by prisms, transferring the strategy he developed for pigments [9]. However, his early work on reflected colour can be appreciated better when placed against a backdrop derived from measurements typifying current research and teaching. The bonus is that it brings to the fore the often overlooked fact that as well as a renowned theorist James Clerk Maxwell was a brilliant experimenter. In 1860, he was awarded the prestigious Rumford Medal of the Royal Society of London, 'for his research on the composition of colours, and other optical properties'.

\section{ACKNOWLEDGEMENTS}

The author gratefully acknowledges the use of facilities in the School of Physics and Astronomy, Edinburgh University, and items from the archives of the James Clerk Maxwell Foundation, 14 India Street, Edinburgh (Maxwell's birthplace in 1831). 


\section{REFERENCES}

[1] Young, T., The Bakerian lecture: on the theory of light and colours. Phil. Trans. R. Soc. London, 92, pp. 12-48, 1802. doi:10.1098/rstl.1802.0004

[2] Coote, J.H., An Illustrated History of Colour Photography, Fountain Press, 1993.

[3] Maxwell, J.C., Experiments on colour, as perceived by the eye, with remarks on colour blindness. Trans. Roy. Soc. Edin., 21, pp. 275-298, 1857.

[4] Newton, I., Optiks, Dover Publications Inc.: New York, 1952.

[5] Campbell, L. \& Garnett, W., Life of James Clerk Maxwell, Macmillan \& Co: London, 1882.

[6] http://www.colour.org.uk/xydiagram.png.

[7] Meyn, J.-P., Colour mixing based on daylight. Eur J Phys, 29, pp. 1017-1032, 2008. doi:10.1088/0143-0807/29/5/014

[8] Erjavac, M. \& Vaupotic, N., Bottle model of colour vision with the colour brown as an example. Eur. J. Phys., 27, pp. 611-620, 2006. doi:10.1088/0143-0807/27/3/013

[9] Dougal, R.C., Greated, C.A. \& Marson, A.J., Then and now: James Clerk Maxwell and colour. Optic Laser Tech., 38, pp. 210-218, 2006. doi:10.1016/j.optlastec.2005.06.036 